\title{
Forms and Distribution of Potassium in Selected Maize Growing Soils of Haveri District of Karnataka, India
}

\author{
B.R. Harsha and B.R. Jagadeesh* \\ Department of Soil Science and Agricultural Chemistry, UAS (Dharwad), College of \\ Agriculture, Hanumanamatti -581115, Haveri District, Karnataka, India \\ *Corresponding author
}

\section{A B S T R A C T}

\begin{tabular}{|l|}
\hline Ke y w o r d s \\
Potassium, \\
Lattice K, Soil \\
reaction, Clay, \\
Exchangeable \\
cations.
\end{tabular}

Potassium an important major nutrient exists in soil as different forms and these forms are in a state of quasi-equilibrium with each other. These different forms of potassium and their distribution were studied in twenty representative red and black soil types spreading over seven taluks of Haveri district of Karnataka. Soils were generally sandy clay loam to clay in texture. The soil reaction ranged between slightly acidic and near neutral in reaction and electrical conductivity was well within the permissible limit. The organic carbon content was low to medium. The average CEC, potassium adsorption ratio, sum of exchangeable cations and available potassium of black soils was high compared to red soils at both the depths. Results of the study indicated that all the forms of potassium found higher in black soils compared to red soils. The water soluble and exchangeable $\mathrm{K}$ was higher in surface sample compared to sub surface in both the soil type. Whereas, nonexchangeable, lattice and total $\mathrm{K}$ was higher in sub surface than the surface in both the soil types. The water soluble form of $\mathrm{K}$ showed a significant and positive correlation with sand in red $\left(\mathrm{r}=0.755^{*}\right)$ and black $\left(\mathrm{r}=0.527^{*}\right)$ soils. The exchangeable $\mathrm{K}$ was significantly and positively correlated with $\mathrm{CEC}\left(\mathrm{r}=0.721^{*}\right.$ and $\mathrm{r}=0.807^{*}$ in red and black soils, respectively) whereas, non-exchangeable $\mathrm{K}$, lattice $\mathrm{K}$ and total $\mathrm{K}$ showed positive correlation for clay.

\section{Introduction}

The biotic cycling of potassium $(\mathrm{K})$ in crop land systems has been relatively understudied in comparison with nitrogen $(\mathrm{N})$ and phosphorus (P) despite its critical roles in maintaining the nutrition of primary production in crop plants. Maize growing soils in India commonly have high levels of potassium down to depths of more than one meter (Datta, 2011). Historically high removal rates by crops such as maize and cotton have depleted soil $\mathrm{K}$ pools and $\mathrm{K}$ deficiencies have been observed in crops.
Many tropical and subtropical soils are poor in mobile compounds of phosphorus, nitrogen and to a lesser extent potassium. The potassium content in tropical soils differ depending on the extent of weathering of their mineral part; the greater is the level of weathering, the lower is the content of potassium in the soil.

Potassium in soil can be allocated to three pools of availability for uptake by roots. It is dissolved in soil water, adsorbed onto 
particles of clay and organic matter and held within the crystal structures of feldspar and mica. The organic matter in soils contains a negligible amount of $\mathrm{K}$ because it is not a constituent of biomolecule, hence is easily and quickly leached from leaves due to its high solubility. However, the fraction of soil $\mathrm{K}$ directly available to plants (i.e., in soil solution) is usually a small proportion (0.100.20 per cent) of the total soil $\mathrm{K}$, whereas both the immediately available water-soluble fraction and the exchangeable fraction comprise 1.00-2.00 per cent and the soilunavailable fractions 96-99 per cent (Britzke et al., 2012).

Potash fertilizers are not effective for all soils and therefore before their application it is necessary to assess the amount of potassium in the soils which is to be fertilized and the degree of availability to plant. The $\mathrm{K}$ fertilization should be based on knowledge of quantity of $\mathrm{K}$ in various types and sub types of soils and not on general specifications based on crop requirement. More fertilizers probably used for maize than for any other annual crop, and this in turn have given rise to new basic problems not considered previously. In Karnataka, the maize growing area is increasing rapidly, adoption of nutrient management practices in general and $\mathrm{K}$ nutrient management in particular is need of the hour. Hence, an immense study was under taken on $\mathrm{K}$ dynamics under maize cropping system in two soil types representing the major soils of Haveri district.

\section{Materials and Methods}

The surface $(0-20 \mathrm{~cm})$ and sub-surface (20-50 $\mathrm{cm})$ soil samples were collected from fourty location belonging to seven taluks of maize growing areas in Haveri district of Karnataka. The soil samples were processed and analysed for $\mathrm{pH}$ (1:2.5) (Jackson, 1973), EC (1:2.5) (Jackson, 1973), OC by Walkley and Black's wet oxidation method (Piper 1996), CEC (Jackson, 1973), available potassium (Jackson, 1973), exchangeable cations (Jackson, 1973), EPP and PAR (U.S.D.A, 1954), particle size distribution (Jackson, 1973), water soluble K (1:5) (Black, 1965), exchangeable potassium was determined by extracting with $N N \mathrm{NH}_{4} \mathrm{OAc}$ solution as outlined by Knudsen et al., (1982). The water-soluble $\mathrm{K}$ was subtracted from $\mathrm{NH}_{4} \mathrm{OAc}-\mathrm{K}$ to get the exchangeable potassium content of the soil. The nonexchangeable $\mathrm{K}$ was estimated by boiling $1 N$ $\mathrm{HNO}_{3}$ method as outlined by Knudsen et al., (1982). The quantity of $\mathrm{K}$ obtained with the $\mathrm{NH}_{4} \mathrm{OAc}$ extract was subtracted to get the non-exchangeable potassium content in the soil. Total potassium content was determined by digesting the samples with hydrofluoric acid in a closed vessel (Lim and Jackson, 1982). The lattice potassium was computed as difference between total potassium and the sum of water soluble, exchangeable and nonexchangeable $\mathrm{K}$ fractions (Tables 1 and 2).

\section{Results and Discussion}

\section{Physico-chemical properties of soil}

The $\mathrm{pH}$ varied from slightly acidic to normal (6.38 to 7.85 ) in reaction. The red soils were having low $\mathrm{pH}$ (6.38) compared to black soils (Tables 3 and 4). In both the soil types, $\mathrm{pH}$ of sub surface samples was high compared to surface. The electrical conductivity in both the soil types was well within the permissible limits $\left(<1.0 \mathrm{dSm}^{-1}\right)$. In both the soil types, EC increased with depth. The organic carbon content varied from low $\left(4.50 \mathrm{~g} \mathrm{mg}^{-1}\right)$ to high $\left(7.98 \mathrm{~g} \mathrm{mg}^{-1}\right)$. The organic carbon content in surface samples of black soil was high compared to red soils. The texture of soil varied from sandy clay loam to clay loam in texture for both the depths in red soils while that of black soils were clay loam to clay in texture in surface samples and clay in sub 
surface samples. The average CEC of black soils in surface layer was high $(38.53 \mathrm{cmol}$ $\left.\left(\mathrm{p}^{+}\right) \mathrm{kg}^{-1}\right)$ compared to red soils $(20.72 \mathrm{cmol}$ $\left(\mathrm{p}^{+}\right) \mathrm{kg}^{-1}$ ) (Table 5 and 6). In case of sub surface depth, average CEC of higher value was noticed in black soils $\left(41.58 \mathrm{cmol}\left(\mathrm{p}^{+}\right)\right.$ $\left.\mathrm{kg}^{-1}\right)$ compared to red soils $\left(23.62 \mathrm{cmol}\left(\mathrm{p}^{+}\right)\right.$ $\left.\mathrm{kg}^{-1}\right)$. The average values of sum of exchangeable cations was higher in black soils $\left(35.64 \mathrm{cmol}\left(\mathrm{p}^{+}\right) \quad \mathrm{kg}^{-1}\right)$ and 38.84 $\operatorname{cmol}\left(\mathrm{p}^{+}\right) \mathrm{kg}^{-1}$ in surface and sub-surface layers, respectively) compared to red soils $\left(18.55 \mathrm{cmol}\left(\mathrm{p}^{+}\right) \mathrm{kg}^{-1}\right.$ and $22.07 \mathrm{cmol}\left(\mathrm{p}^{+}\right) \mathrm{kg}^{-1}$ in surface and sub-surface depths). The calcium and magnesium were dominant cation in both the soil type. The average values of exchangeable potassium percentage were high in red soils at both the depths (11.38 and 8.50 per cent in surface and subsurface depths, respectively). The average value of potassium adsorption ratio was high in black soils at both the depths (2.25 and 1.34 in surface and sub-surface depths, respectively). The available potassium on an average obtained highest in black soils at both the depths $\left(529.53 \mathrm{~kg} \mathrm{ha}^{-1}\right.$ and $327.04 \mathrm{~kg} \mathrm{ha}^{-1}$ in surface and sub-surface depths, respectively) when compared to red soils (339.53 kg ha ${ }^{-1}$ and $281.44 \mathrm{~kg} \mathrm{ha}^{-1}$ surface and sub-surface depths, respectively). In both the soil types, surface samples recorded high available potassium compared to sub surface.

\section{Forms and distribution of potassium}

The data related to forms and distribution of potassium in surface and sub-surface depths of red and black soils are presented in tables 7 and 8 , respectively.

\section{Water soluble K}

The water soluble potassium in selected red soils of maize growing areas of Haveri district varied from 1.66 to $4.69 \mathrm{mg} \mathrm{kg}^{-1}$ with a mean of $2.91 \mathrm{mg} \mathrm{kg}^{-1}$ in surface depth (Table 7). The sub surface water soluble $\mathrm{K}$ content varied from 1.31 to $3.39 \mathrm{mg} \mathrm{kg}^{-1}$ with a mean of $2.24 \mathrm{mg} \mathrm{kg}^{-1}$. The water soluble $\mathrm{K}$ of black soils ranged from 2.83 to $8.13 \mathrm{mg} \mathrm{kg}^{-1}$ in surface with a mean of $4.60 \mathrm{mg} \mathrm{kg}^{-1}$. The water soluble potassium in sub surface layer varied from 2.00 to $6.23 \mathrm{mg} \mathrm{kg}^{-1}$ with a mean of $3.67 \mathrm{mg} \mathrm{kg}^{-1}$ (Table 8). The black soils recorded more water soluble $\mathrm{K}$ at surface as well as sub surface depths compared to red soils was due to the fact that black soils have greater moisture retention capacity and nutrient holding capacity than red soils and also organic matter content was relatively higher in these soils (Anil et al., 2009).

\section{Exchangeable K}

The exchangeable potassium content of red soils ranged from 44.80 to $78.40 \mathrm{mg} \mathrm{kg}^{-1}$ in surface layer. The sub surface exchangeable potassium content of red soils varied from 30.60 to $67.20 \mathrm{mg} \mathrm{kg}^{-1}$. The mean value of exchangeable $\mathrm{K}$ was 52.28 and $45.24 \mathrm{mg} \mathrm{kg}^{-1}$ in surface and sub-surface layers, respectively. The exchangeable potassium in black soils varied from 48.92 to $100.96 \mathrm{mg}$ $\mathrm{kg}^{-1}$ in surface and 36.90 to $78.40 \mathrm{mg} \mathrm{kg}^{-1}$ in sub surface. The mean content of exchangeable $\mathrm{K}$ was 56.00 and $46.81 \mathrm{mg} \mathrm{kg}^{-1}$ in surface and sub-surface samples, respectively.

The mean exchangeable potassium of black soils were high compared to red soils it may be due to fact that black soils are rich in organic matter content and in general, dominated by 2:1 type of clay minerals which offered more exchange sites for $\mathrm{K}$. The results were in corroboration with that of Anil et al., (2009), Gali and Hebsur (2011) (Fig. 1).

\section{Non-exchangeable $\mathrm{K}$}

The average non-exchangeable potassium in surface soils of red soil type recorded 581.53 $\mathrm{mg} \mathrm{kg}^{-1}$ and that of sub surface layer recorded $723.62 \mathrm{mg} \mathrm{kg}^{-1}$. 
Table.1 Details of soil samples collected from different places (red type) of maize growing areas of Haveri district, Karnataka

\begin{tabular}{|c|c|c|c|c|}
\hline Sl. No. & Taluk & Location & Latitude & Longitude \\
\hline 1 & Shiggoan & Kengapur-1 & $15^{\circ} 0223.0^{\prime \prime}$ & $74^{\circ} 59^{\prime} 13.1^{\prime \prime}$ \\
\hline 2 & Shiggoan & Kengapur-2 & $15^{\circ} 0240.3^{\prime \prime}$ & $75^{\circ} 15^{\prime} 37.3^{\prime \prime}$ \\
\hline 3 & Shiggoan & Kengapur-3 & $15^{\circ} 0229.7^{\prime \prime}$ & $75^{\circ} 15^{\prime} 35.2^{\prime}$ \\
\hline 4 & Shiggoan & Hulgur-1 & $15^{\circ} 0115.1^{\prime \prime}$ & $75^{\circ} 18 \quad 42.0^{\prime \prime}$ \\
\hline 5 & Shiggoan & Hulgur-2 & $15^{\circ} 02^{\prime} 25.5^{\prime}$ & $75^{\circ} 15^{\prime} 55.2^{\prime \prime}$ \\
\hline 6 & Savanur & Savanur & $14^{\circ} 53^{\prime} 55.2^{\prime}$ & $75^{\circ} 20^{\prime} 31.3^{\prime \prime}$ \\
\hline 7 & Haveri & Guttal & $14^{\circ} 5812.2^{\prime \prime}$ & $75^{\circ} 2422.8^{\prime \prime}$ \\
\hline 8 & Ranebennur & Aremallapur-1 & $14^{\circ} 59^{\prime} 18.0^{\prime \prime}$ & $75^{\circ} 6032.0^{\prime}$ \\
\hline 9 & Ranebennur & Aremallapur-2 & $14^{\circ} 5920.0^{\prime \prime}$ & $75^{\circ} 60 \quad 38.0^{\prime \prime}$ \\
\hline 10 & Ranebennur & Aremallapur-3 & $14^{\circ} 59^{\prime} 38.1^{\prime \prime}$ & $75^{\circ} 60^{\prime} 35.1^{\prime \prime}$ \\
\hline 11 & Ranebennur & Aremallapur-4 & $14^{\circ} 59^{\prime} 38.2^{\prime \prime}$ & $75^{\circ} 60^{\prime} 31.3^{\prime}$ \\
\hline 12 & Ranebennur & Aremallapur-5 & $14^{\circ} 5917.0^{\prime \prime}$ & $75^{\circ} 60 \quad 32.8^{\prime \prime}$ \\
\hline 13 & Ranebennur & Medleri-1 & $14^{\circ} 59^{\prime} 19.9^{\prime \prime}$ & $75^{\circ} 60^{\prime} 42.0^{\prime \prime}$ \\
\hline 14 & Ranebennur & Medleri-2 & $14^{\circ} 59^{\prime} 57.2^{\prime}$ & $75^{\circ} 6048.0^{\prime \prime}$ \\
\hline 15 & Ranebennur & Medleri-3 & $14^{\circ} 5859.1^{\prime \prime}$ & $75^{\circ} 60 \quad 35.1^{\prime \prime}$ \\
\hline 16 & Ranebennur & Medleri-4 & $14^{\circ} 5837.1^{\prime \prime}$ & $75^{\circ} 60 \quad 39.0^{\prime \prime}$ \\
\hline 17 & Ranebennur & Medleri-5 & $14^{\circ} 58^{\prime} 36.2^{\prime \prime}$ & $75^{\circ} 59^{\prime} 46.2^{\prime \prime}$ \\
\hline 18 & Byadgi & Byadgi-1 & $14^{\circ} 49^{\prime} 54.0^{\prime \prime}$ & $75^{\circ} 46^{\prime} 42.1^{\prime \prime}$ \\
\hline 19 & Byadgi & Byadgi-2 & $14^{\circ} 44^{\prime} 15.3^{\prime \prime}$ & $75^{\circ} 46 \quad 49.0^{\prime \prime}$ \\
\hline 20 & Ranebennur & Hanumanamatti & $14^{\circ} 59^{\prime} 11.8^{\prime \prime}$ & $75^{\circ} 39^{\prime} 44.8^{\prime \prime}$ \\
\hline
\end{tabular}

Table.2 Details of soil samples collected from different places (black type) of maize growing areas of Haveri district, Karnataka

\begin{tabular}{|c|c|c|c|c|}
\hline Sl. No. & Taluk & Location & Latitude & Longitude \\
\hline 1 & Shiggoan & Bankapur-1 & $15^{\circ} 0252.1^{\prime \prime}$ & $75^{\circ} 15^{\prime} 16.1^{\prime \prime}$ \\
\hline 2 & Shiggoan & Bankapur-2 & $15^{\circ} 02^{\prime} 20.5^{\prime}$ & $75^{\circ} 15^{\prime} 55.0^{\prime \prime}$ \\
\hline 3 & Savanur & Kengapur & $15^{\circ} 01^{\prime} 21.3^{\prime \prime}$ & $75^{\circ} 14^{\prime} 40.0^{\prime \prime}$ \\
\hline 4 & Savanur & Mannangi-1 & $14^{\circ} 5305.3^{\prime \prime}$ & $75^{\circ} 17^{\prime} 39.7^{\prime \prime}$ \\
\hline 5 & Savanur & Mannangi-2 & $14^{\circ} 5258.7^{\prime \prime}$ & $75^{\circ} 17^{\prime} 37.7^{\prime \prime}$ \\
\hline 6 & Savanur & Savoor & $14^{\circ} 5342.8^{\prime \prime}$ & $75^{\circ} 17^{\prime} 28.0^{\prime \prime}$ \\
\hline 7 & Haveri & Haveri & $14^{\circ} 53$ '45.0”, & $75^{\circ} 27^{\prime} 54.1^{\prime \prime}$ \\
\hline 8 & Haveri & Devihosur & $14^{\circ} 53^{\prime} 40.4^{\prime}$ & $75^{\circ} 29^{\prime} 41.7^{\prime}$ \\
\hline 9 & Hangal & Hangal & $14^{\circ} 59^{\prime} 13.2^{\prime \prime}$ & $75^{\circ} 57^{\prime} 32.5^{\prime}$ \\
\hline 10 & Byadgi & Kaginelli & $14^{\circ} 51^{\prime} 28.2^{\prime \prime}$ & $75^{\circ} 44^{\prime} 32.0^{\prime \prime}$ \\
\hline 11 & Hirekerur & Rattihalli-1 & $14^{\circ} 51^{\prime} 18.2^{\prime \prime}$ & $75^{\circ} 44^{\prime} 32.0^{\prime \prime}$ \\
\hline 12 & Hirekerur & Rattihalli-2 & $14^{\circ} 51^{\prime} 36.3^{\prime \prime}$ & $75^{\circ} 39^{\prime} 36.1^{\prime \prime}$ \\
\hline 13 & Hirekerur & Rattihalli-3 & $14^{\circ} 51^{\prime} 21.0^{\prime \prime}$ & $75^{\circ} 33^{\prime} 39.4^{\prime \prime}$ \\
\hline 14 & Hirekerur & Rattihalli-4 & $14^{\circ} 52^{\prime} 29.9^{\prime \prime}$ & $75^{\circ} 33^{\prime} 40.2^{\prime \prime}$ \\
\hline 15 & Hirekerur & Rattihalli-5 & $14^{\circ} 49^{\prime} 32.0^{\prime \prime}$ & $75^{\circ} 33^{\prime} 42.0^{\prime}$ \\
\hline 16 & Hirekerur & Rattihalli-6 & $14^{\circ} 49^{\prime} 34.2^{\prime \prime}$ & $75^{\circ} 33^{\prime} 46.0^{\prime \prime}$ \\
\hline 17 & Hirekerur & Jogihalli-1 & $14^{\circ} 45^{\prime} 10.6^{\prime \prime}$ & $75^{\circ} 39^{\prime} 36.8^{\prime \prime}$ \\
\hline 18 & Hirekerur & Jogihalli-2 & $14^{\circ} 52^{\prime} 48.8^{\prime \prime}$ & $75^{\circ} 30^{\prime} 33.0^{\prime \prime}$ \\
\hline 19 & Hirekerur & Jogihalli-3 & $14^{\circ} 52^{\prime} 52.1^{\prime \prime}$ & $75^{\circ} 30^{\prime} 32.2^{\prime \prime}$ \\
\hline 20 & Hirekerur & Jogihalli-4 & $14^{\circ} 45^{\prime} 14.0^{\prime \prime}$ & $75^{\circ} 39^{\prime} 11.9^{\prime \prime}$ \\
\hline
\end{tabular}


Table.3 Physico-chemical properties in selected maize growing red soils of Haveri district

\begin{tabular}{|c|c|c|c|c|c|c|c|c|}
\hline \multirow[b]{2}{*}{$\begin{array}{c}\text { Sample } \\
\text { No. }\end{array}$} & \multicolumn{2}{|c|}{$\mathrm{pH}_{1: 2.5}$} & \multicolumn{2}{|c|}{$\begin{array}{c}E C_{1: 2.5} \\
\left(d S ~ m^{-1}\right)\end{array}$} & \multicolumn{2}{|c|}{$\begin{array}{c}\mathrm{OC} \\
\left(\mathrm{g} \mathrm{kg}^{-1}\right)\end{array}$} & \multicolumn{2}{|c|}{ Textural class } \\
\hline & $\begin{array}{l}\text { Surface } \\
(0-20 \mathrm{~cm})\end{array}$ & $\begin{array}{c}\text { Sub } \\
\text { surface } \\
(20-50 \\
\text { cm) }\end{array}$ & $\begin{array}{c}\text { Surface } \\
(0-20 \\
\text { cm }) \\
\end{array}$ & $\begin{array}{c}\text { Sub } \\
\text { surface } \\
(20-50 \mathrm{~cm})\end{array}$ & $\begin{array}{l}\text { Surface } \\
(0-20 \mathrm{~cm})\end{array}$ & $\begin{array}{c}\text { Sub } \\
\text { surface } \\
(20-50 \mathrm{~cm})\end{array}$ & $\begin{array}{l}\text { Surface } \\
(0-20 \mathrm{~cm})\end{array}$ & $\begin{array}{c}\text { Sub } \\
\text { Surface } \\
(20-50 \mathrm{~cm})\end{array}$ \\
\hline 1 & 6.59 & 6.53 & 0.05 & 0.07 & 6.04 & 4.23 & Scl & Scl \\
\hline 2 & 6.75 & 6.77 & 0.06 & 0.04 & 4.50 & 3.12 & Scl & Scl \\
\hline 3 & 6.50 & 6.55 & 0.03 & 0.03 & 5.01 & 4.47 & Scl & $\mathrm{Cl}$ \\
\hline 4 & 6.70 & 6.74 & 0.04 & 0.05 & 5.72 & 3.98 & Scl & Scl \\
\hline 5 & 6.66 & 6.67 & 0.03 & 0.04 & 5.35 & 4.56 & Scl & Scl \\
\hline 6 & 7.50 & 7.52 & 0.25 & 0.27 & 5.20 & 4.41 & Scl & Scl \\
\hline 7 & 6.68 & 6.70 & 0.03 & 0.05 & 4.63 & 4.01 & Scl & Scl \\
\hline 8 & 6.50 & 6.53 & 0.05 & 0.06 & 5.12 & 3.97 & Scl & Scl \\
\hline 9 & 6.75 & 6.79 & 0.04 & 0.05 & 5.78 & 4.32 & $\mathrm{Cl}$ & $\mathrm{Cl}$ \\
\hline 10 & 7.36 & 7.37 & 0.19 & 0.21 & 6.11 & 5.25 & Scl & Scl \\
\hline 11 & 7.30 & 7.32 & 0.18 & 0.18 & 5.07 & 4.33 & Scl & Scl \\
\hline 12 & 7.40 & 7.43 & 0.18 & 0.20 & 5.19 & 4.86 & $\mathrm{Cl}$ & $\mathrm{Cl}$ \\
\hline 13 & 7.41 & 7.45 & 0.09 & 0.12 & 6.40 & 4.98 & Scl & Scl \\
\hline 14 & 6.68 & 6.70 & 0.11 & 0.14 & 5.10 & 4.32 & Scl & $\mathrm{Cl}$ \\
\hline 15 & 7.24 & 7.25 & 0.22 & 0.23 & 6.20 & 5.11 & Scl & Scl \\
\hline 16 & 6.55 & 6.54 & 0.06 & 0.09 & 4.62 & 3.14 & Scl & $\mathrm{Cl}$ \\
\hline 17 & 6.38 & 6.37 & 0.07 & 0.07 & 6.90 & 4.47 & Scl & Scl \\
\hline 18 & 7.08 & 7.10 & 0.06 & 0.08 & 7.90 & 6.18 & Scl & Scl \\
\hline 19 & 7.00 & 7.05 & 0.05 & 0.05 & 7.00 & 5.98 & Scl & $\mathrm{Cl}$ \\
\hline 20 & 7.46 & 7.47 & 0.11 & 0.09 & 6.50 & 5.27 & $\mathrm{Cl}$ & $\mathrm{Cl}$ \\
\hline Range & 6.38-7.50 & $6.37-7.52$ & 0.03-0.25 & 0.03-0.27 & $4.50-7.90$ & 3.12-6.18 & Sandy clay & Sandy clay \\
\hline Mean & 6.92 & 6.94 & 0.09 & 0.11 & 5.72 & 4.54 & loam to clay & loam to clay \\
\hline S.D. & 0.380 & 0.386 & 0.069 & 0.073 & 0.901 & 0.780 & loam & loam \\
\hline
\end{tabular}

Scl- Sandy clay loam, Cl- Clay loam 
Table.4 Physico-chemical properties in selected maize growing black soils of Haveri district

\begin{tabular}{|c|c|c|c|c|c|c|c|c|}
\hline \multirow[b]{2}{*}{$\begin{array}{c}\text { Sample } \\
\text { No. }\end{array}$} & \multicolumn{2}{|c|}{$\mathbf{p H}_{1: 2.5}$} & \multicolumn{2}{|c|}{$\begin{array}{c}\mathrm{EC}_{1: 2.5} \\
\left(\mathrm{dS} \mathrm{\textrm {m } ^ { - 1 }}\right)\end{array}$} & \multicolumn{2}{|c|}{$\begin{array}{c}\mathrm{OC} \\
\left(\mathrm{g} \mathrm{kg}^{-1}\right)\end{array}$} & \multicolumn{2}{|c|}{ Textural class } \\
\hline & $\begin{array}{c}\text { Surface } \\
(0-20 \mathrm{~cm})\end{array}$ & $\begin{array}{c}\text { Sub } \\
\text { surface }\end{array}$ & $\begin{array}{l}\text { Surface } \\
(0-20 \mathrm{~cm})\end{array}$ & $\begin{array}{c}\text { Sub } \\
\text { surface } \\
(20-50 \\
\text { cm) }\end{array}$ & $\begin{array}{l}\text { Surface } \\
(0-20 \mathrm{~cm})\end{array}$ & $\begin{array}{c}\text { Sub } \\
\text { surface } \\
(20-50 \mathrm{~cm})\end{array}$ & $\begin{array}{c}\text { Surface } \\
(0-20 \\
\text { cm) }\end{array}$ & $\begin{array}{c}\text { Sub } \\
\text { surface } \\
(20-50 \mathrm{~cm})\end{array}$ \\
\hline 1 & 7.60 & 7.58 & 0.22 & 0.25 & 5.94 & 5.14 & $\mathrm{C}$ & $\mathrm{C}$ \\
\hline 2 & 7.20 & 7.22 & 0.26 & 0.27 & 6.20 & 5.71 & $\mathrm{Cl}$ & $\mathrm{C}$ \\
\hline 3 & 7.40 & 7.36 & 0.28 & 0.27 & 4.88 & 3.65 & $\mathrm{C}$ & $\mathrm{C}$ \\
\hline 4 & 7.00 & 7.02 & 0.35 & 0.36 & 6.41 & 6.14 & $\mathrm{C}$ & $\mathrm{C}$ \\
\hline 5 & 7.77 & 7.80 & 0.26 & 0.29 & 6.64 & 5.23 & $\mathrm{Cl}$ & $\mathrm{C}$ \\
\hline 6 & 7.35 & 7.40 & 0.25 & 0.30 & 7.12 & 6.02 & $\mathrm{C}$ & $\mathrm{C}$ \\
\hline 7 & 7.68 & 7.70 & 0.28 & 0.33 & 7.01 & 6.21 & $\mathrm{Cl}$ & $\mathrm{C}$ \\
\hline 8 & 7.85 & 7.77 & 0.26 & 0.25 & 7.14 & 6.28 & $\mathrm{C}$ & $\mathrm{C}$ \\
\hline 9 & 7.38 & 7.40 & 0.28 & 0.32 & 7.98 & 6.45 & $\mathrm{C}$ & $\mathrm{C}$ \\
\hline 10 & 7.48 & 7.52 & 0.33 & 0.35 & 7.01 & 6.23 & $\mathrm{C}$ & $\mathrm{C}$ \\
\hline 11 & 7.62 & 7.61 & 0.27 & 0.30 & 6.14 & 5.19 & $\mathrm{C}$ & $\mathrm{C}$ \\
\hline 12 & 7.65 & 7.61 & 0.29 & 0.33 & 6.47 & 5.35 & $\mathrm{C}$ & $\mathrm{C}$ \\
\hline 13 & 7.60 & 7.58 & 0.32 & 0.31 & 6.24 & 5.11 & $\mathrm{C}$ & $\mathrm{C}$ \\
\hline 14 & 7.61 & 7.64 & 0.30 & 0.32 & 6.98 & 5.98 & $\mathrm{C}$ & $\mathrm{C}$ \\
\hline 15 & 7.63 & 7.64 & 0.29 & 0.31 & 7.41 & 6.35 & $\mathrm{C}$ & $\mathrm{C}$ \\
\hline 16 & 7.54 & 7.55 & 0.31 & 0.30 & 6.66 & 5.29 & $\mathrm{C}$ & $\mathrm{C}$ \\
\hline 17 & 7.65 & 7.66 & 0.25 & 0.25 & 7.23 & 6.35 & $\mathrm{C}$ & $\mathrm{C}$ \\
\hline 18 & 7.61 & 7.62 & 0.30 & 0.32 & 7.08 & 6.01 & $\mathrm{C}$ & $\mathrm{C}$ \\
\hline 19 & 7.58 & 7.60 & 0.27 & 0.29 & 7.22 & 6.03 & $\mathrm{C}$ & $\mathrm{C}$ \\
\hline 20 & 7.58 & 7.61 & 0.30 & 0.34 & 7.96 & 6.39 & $\mathrm{C}$ & $\mathrm{C}$ \\
\hline Range & $7.00-7.85$ & 7.02-7.80 & $0.22-0.35$ & $0.25-0.36$ & 4.88-7.98 & $3.65-6.45$ & Clay & \\
\hline Mean & $\mathbf{7 . 5 3}$ & 7.54 & 0.28 & 0.31 & 6.78 & 5.70 & loam to & Clay \\
\hline S.D. & 0.19 & 0.18 & 0.031 & 0.035 & 0.72 & 0.69 & clay & \\
\hline
\end{tabular}

$\mathrm{Cl}$ - Clay loam, C- Clay 
Table.5 Cation exchange capacity, Exchangeable potassium percentage, Potassium adsorption ratio, Sum of exchangeable cations and available Potassium in selected maize growing red soils of Haveri district

\begin{tabular}{|c|c|c|c|c|c|c|c|c|c|c|}
\hline \multirow{2}{*}{$\begin{array}{l}\text { Sample } \\
\text { No. }\end{array}$} & \multicolumn{2}{|c|}{$\begin{array}{c}\text { CEC } \\
\left(\operatorname{cmol}\left(\mathbf{p}^{+}\right) \mathrm{kg}^{-1}\right)\end{array}$} & \multicolumn{2}{|c|}{$\begin{array}{c}\text { Exchangeable } \\
\text { potassium percentage } \\
(\%)\end{array}$} & \multicolumn{2}{|c|}{$\begin{array}{c}\text { Potassium adsorption } \\
\text { ratio }\end{array}$} & \multicolumn{2}{|c|}{$\begin{array}{c}\text { Sum of exchangeable } \\
\text { cations } \\
\left(\mathrm{cmol}^{+}\left(\mathrm{p}^{+}\right) \mathrm{kg}^{-1}\right) \\
\end{array}$} & \multicolumn{2}{|c|}{$\begin{array}{l}\text { Available } \mathrm{K}_{2} \mathrm{O} \\
\quad\left(\mathrm{kg} \mathrm{ha}^{-1}\right)\end{array}$} \\
\hline & $\begin{array}{l}\text { Surface } \\
(0-20 \mathrm{~cm})\end{array}$ & $\begin{array}{c}\text { Sub } \\
\text { surface } \\
(20-50 \mathrm{~cm}) \\
\end{array}$ & $\begin{array}{l}\text { Surface } \\
(0-20 \mathrm{~cm})\end{array}$ & $\begin{array}{c}\text { Sub } \\
\text { surface } \\
(20-50 \mathrm{~cm}) \\
\end{array}$ & $\begin{array}{l}\text { Surface } \\
(0-20 \mathrm{~cm})\end{array}$ & $\begin{array}{c}\text { Sub } \\
\text { surface } \\
(20-50 \mathrm{~cm}) \\
\end{array}$ & $\begin{array}{l}\text { Surface } \\
(0-20 \mathrm{~cm})\end{array}$ & $\begin{array}{c}\text { Sub } \\
\text { surface } \\
(20-50 \mathrm{~cm}) \\
\end{array}$ & $\begin{array}{l}\text { Surface } \\
(0-20 \mathrm{~cm})\end{array}$ & $\begin{array}{c}\text { Sub } \\
\text { surface } \\
(20-50 \mathrm{~cm}) \\
\end{array}$ \\
\hline 1 & 15.21 & 19.26 & 14.33 & 10.69 & 2.23 & 1.73 & 14.19 & 17.75 & 328.56 & 268.82 \\
\hline 2 & 19.34 & 25.98 & 12.92 & 8.12 & 1.91 & 0.74 & 18.01 & 25.44 & 376.32 & 256.20 \\
\hline 3 & 23.65 & 26.48 & 8.75 & 7.43 & 1.08 & 0.90 & 20.83 & 24.96 & 274.30 & 228.80 \\
\hline 4 & 19.36 & 20.45 & 10.95 & 9.33 & 1.94 & 1.69 & 17.93 & 18.20 & 321.12 & 272.40 \\
\hline 5 & 16.92 & 19.37 & 12.47 & 8.01 & 0.89 & 0.63 & 15.36 & 18.67 & 271.80 & 225.24 \\
\hline 6 & 21.86 & 24.89 & 9.88 & 6.38 & 1.51 & 0.81 & 19.84 & 23.40 & 312.96 & 237.42 \\
\hline 7 & 24.15 & 25.94 & 14.49 & 9.63 & 2.98 & 1.90 & 22.59 & 24.07 & 483.84 & 376.32 \\
\hline 8 & 16.88 & 20.31 & 12.14 & 7.48 & 0.89 & 1.01 & 15.24 & 19.44 & 302.16 & 258.60 \\
\hline 9 & 26.32 & 27.78 & 8.32 & 7.23 & 2.12 & 1.94 & 23.85 & 25.46 & 322.56 & 298.20 \\
\hline 10 & 16.22 & 19.32 & 15.41 & 11.95 & 2.67 & 1.41 & 14.64 & 18.21 & 430.08 & 321.56 \\
\hline 11 & 22.76 & 24.97 & 9.27 & 7.20 & 1.10 & 1.03 & 19.80 & 24.41 & 268.80 & 215.04 \\
\hline 12 & 27.05 & 28.92 & 9.24 & 8.50 & 1.65 & 1.21 & 24.13 & 27.00 & 430.08 & 366.38 \\
\hline 13 & 18.43 & 21.22 & 11.93 & 7.49 & 1.85 & 0.84 & 16.27 & 19.37 & 341.26 & 295.04 \\
\hline 14 & 20.29 & 24.68 & 10.74 & 8.14 & 1.37 & 1.03 & 18.70 & 23.92 & 302.54 & 261.35 \\
\hline 15 & 17.21 & 19.23 & 13.07 & 10.41 & 1.38 & 0.87 & 15.22 & 18.22 & 323.51 & 271.80 \\
\hline 16 & 24.83 & 27.54 & 8.17 & 7.15 & 1.92 & 1.15 & 22.50 & 25.31 & 371.32 & 298.13 \\
\hline 17 & 18.36 & 22.78 & 10.89 & 6.58 & 0.95 & 0.71 & 16.34 & 21.11 & 269.80 & 235.04 \\
\hline 18 & 17.86 & 20.05 & 17.35 & 14.96 & 2.07 & 1.54 & 16.51 & 18.92 & 374.82 & 342.96 \\
\hline 19 & 23.14 & 27.16 & 9.20 & 7.43 & 2.21 & 1.51 & 20.62 & 25.49 & 376.22 & 320.76 \\
\hline 20 & 24.66 & 26.09 & 8.15 & 5.97 & 1.36 & 1.09 & 22.38 & 24.88 & 308.56 & 278.81 \\
\hline Range & $\begin{array}{l}15.21- \\
27.05 \\
\end{array}$ & $\begin{array}{l}19.23- \\
28.92 \\
\end{array}$ & $\begin{array}{l}8.15- \\
17.35 \\
\end{array}$ & $\begin{array}{l}5.97- \\
14.96 \\
\end{array}$ & $\begin{array}{c}0.89- \\
2.98\end{array}$ & $\begin{array}{c}0.63- \\
1.94 \\
\end{array}$ & $\begin{array}{l}14.19- \\
24.13 \\
\end{array}$ & $\begin{array}{l}17.75- \\
27.00 \\
\end{array}$ & $\begin{array}{l}268.80- \\
483.84 \\
\end{array}$ & $\begin{array}{c}215.04- \\
376.32 \\
\end{array}$ \\
\hline Mean & 20.72 & 23.62 & 11.38 & 8.50 & 1.70 & 1.19 & 18.55 & 22.07 & 339.53 & 281.44 \\
\hline S.D. & 3.63 & 3.38 & 2.63 & 2.15 & 0.58 & 0.40 & 3.23 & 3.24 & 59.08 & 46.01 \\
\hline
\end{tabular}


Table.6 Cation exchange capacity, Exchangeable potassium percentage, Potassium adsorption ratio and Sum of exchangeable cations in selected Maize growing black soils of Haveri district

\begin{tabular}{|c|c|c|c|c|c|c|c|c|c|c|}
\hline \multirow{2}{*}{$\begin{array}{l}\text { Sample } \\
\text { No. }\end{array}$} & \multicolumn{2}{|c|}{$\begin{array}{c}\mathrm{CEC} \\
\left(\operatorname{cmol}\left(\mathbf{p}^{+}\right) \mathbf{k g}^{-1}\right)\end{array}$} & \multicolumn{2}{|c|}{$\begin{array}{c}\text { Exchangeable } \\
\text { potassium percentage } \\
(\%)\end{array}$} & \multicolumn{2}{|c|}{$\begin{array}{c}\text { Potassium adsorption } \\
\text { ratio }\end{array}$} & \multicolumn{2}{|c|}{$\begin{array}{c}\text { Sum of exchangeable } \\
\text { cations } \\
\left(\operatorname{cmol}^{\prime}\left(\mathrm{p}^{+}\right) \mathrm{kg}^{-1}\right)\end{array}$} & \multicolumn{2}{|c|}{$\begin{array}{l}\text { Available } \mathrm{K}_{2} \mathrm{O} \\
\quad\left(\mathrm{kg} \mathrm{ha}^{-1}\right)\end{array}$} \\
\hline & $\begin{array}{l}\text { Surface } \\
(0-20 \mathrm{~cm})\end{array}$ & $\begin{array}{c}\text { Sub } \\
\text { surface } \\
(20-50 \mathrm{~cm})\end{array}$ & $\begin{array}{l}\text { Surface } \\
(0-20 \mathrm{~cm})\end{array}$ & $\begin{array}{c}\text { Sub } \\
\text { surface } \\
(20-50 \mathrm{~cm})\end{array}$ & $\begin{array}{l}\text { Surface } \\
(0-20 \mathrm{~cm})\end{array}$ & $\begin{array}{c}\text { Sub } \\
\text { surface } \\
(20-50 \mathrm{~cm})\end{array}$ & $\begin{array}{l}\text { Surface } \\
(0-20 \mathrm{~cm})\end{array}$ & $\begin{array}{c}\text { Sub } \\
\text { Surface } \\
(20-50 \mathrm{~cm})\end{array}$ & $\begin{array}{l}\text { Surface } \\
(0-20 \mathrm{~cm})\end{array}$ & $\begin{array}{c}\text { Sub } \\
\text { surface } \\
(20-50 \mathrm{~cm})\end{array}$ \\
\hline 1 & 44.98 & 47.84 & 14.33 & 10.69 & 2.23 & 1.73 & 14.19 & 17.75 & 430.28 & 344.00 \\
\hline 2 & 27.14 & 30.14 & 12.92 & 8.12 & 1.91 & 0.74 & 18.01 & 25.44 & 426.64 & 268.87 \\
\hline 3 & 35.45 & 38.78 & 8.75 & 7.43 & 1.08 & 0.90 & 20.83 & 24.96 & 452.81 & 324.21 \\
\hline 4 & 40.16 & 42.27 & 10.95 & 9.33 & 1.94 & 1.69 & 17.93 & 18.20 & 483.84 & 313.66 \\
\hline 5 & 23.64 & 26.34 & 12.47 & 8.01 & 0.89 & 0.63 & 15.36 & 18.67 & 376.32 & 298.10 \\
\hline 6 & 42.54 & 45.65 & 9.88 & 6.38 & 1.51 & 0.81 & 19.84 & 23.40 & 420.08 & 319.60 \\
\hline 7 & 24.26 & 27.39 & 14.49 & 9.63 & 2.98 & 1.90 & 22.59 & 24.07 & 483.84 & 368.20 \\
\hline 8 & 42.21 & 46.05 & 12.14 & 7.48 & 0.89 & 1.01 & 15.24 & 19.44 & 489.27 & 313.60 \\
\hline 9 & 41.89 & 44.95 & 8.32 & 7.23 & 2.12 & 1.94 & 23.85 & 25.46 & 322.56 & 224.00 \\
\hline 10 & 33.14 & 35.76 & 15.41 & 11.95 & 2.67 & 1.41 & 14.64 & 18.21 & 432.08 & 366.31 \\
\hline 11 & 44.25 & 46.82 & 9.27 & 7.20 & 1.10 & 1.03 & 19.80 & 24.41 & 645.12 & 448.00 \\
\hline 12 & 42.27 & 44.08 & 9.24 & 8.50 & 1.65 & 1.21 & 24.13 & 27.00 & 645.12 & 494.20 \\
\hline 13 & 32.74 & 36.75 & 11.93 & 7.49 & 1.85 & 0.84 & 16.27 & 19.37 & 591.36 & 443.04 \\
\hline 14 & 38.18 & 42.74 & 10.74 & 8.14 & 1.37 & 1.03 & 18.70 & 23.92 & 537.60 & 413.60 \\
\hline 15 & 41.85 & 44.06 & 13.07 & 10.41 & 1.38 & 0.87 & 15.22 & 18.22 & 722.64 & 488.85 \\
\hline 16 & 42.06 & 43.94 & 8.17 & 7.15 & 1.92 & 1.15 & 22.50 & 25.31 & 698.88 & 403.24 \\
\hline 17 & 41.21 & 44.58 & 10.89 & 6.58 & 0.95 & 0.71 & 16.34 & 21.11 & 591.36 & 358.40 \\
\hline 18 & 43.86 & 47.24 & 17.35 & 14.96 & 2.07 & 1.54 & 16.51 & 18.92 & 483.84 & 393.67 \\
\hline 19 & 42.18 & 43.72 & 9.20 & 7.43 & 2.21 & 1.51 & 20.62 & 25.49 & 596.15 & 463.62 \\
\hline 20 & 46.78 & 52.61 & 8.15 & 5.97 & 1.36 & 1.09 & 22.38 & 24.88 & 752.64 & 592.80 \\
\hline Range & $\begin{array}{l}23.64- \\
46.78\end{array}$ & $\begin{array}{l}26.34- \\
52.61\end{array}$ & $\begin{array}{l}8.15- \\
17.35\end{array}$ & $\begin{array}{l}5.97- \\
14.96\end{array}$ & $\begin{array}{l}0.89- \\
2.98\end{array}$ & $\begin{array}{c}0.63- \\
1.94\end{array}$ & $\begin{array}{l}14.19- \\
24.13\end{array}$ & $\begin{array}{l}17.75- \\
27.00\end{array}$ & $\begin{array}{l}322.56- \\
752.64\end{array}$ & $\begin{array}{c}224.00- \\
592.80\end{array}$ \\
\hline Mean & 38.53 & 41.58 & 11.38 & 8.50 & 1.70 & 1.19 & 18.55 & 22.07 & 529.53 & 327.04 \\
\hline S.D. & 6.88 & 7.00 & 2.63 & 2.15 & 0.58 & 0.40 & 3.23 & 3.24 & 120.65 & 82.35 \\
\hline
\end{tabular}


Table.7 Forms and distribution of potassium in red soil type of selected maize growing areas of Haveri district

\begin{tabular}{|c|c|c|c|c|c|c|c|c|c|c|}
\hline \multirow[b]{2}{*}{$\begin{array}{c}\text { Sample } \\
\text { No. }\end{array}$} & \multicolumn{2}{|c|}{$\begin{array}{c}\text { Water soluble K } \\
\left(\mathrm{mg} \mathrm{kg}^{-1}\right)\end{array}$} & \multicolumn{2}{|c|}{$\begin{array}{c}\text { Exchangeable } \mathrm{K} \\
\left(\mathrm{mg} \mathrm{kg}^{-1}\right)\end{array}$} & \multicolumn{2}{|c|}{$\begin{array}{c}\text { Non-exchangeable } \mathrm{K} \\
\left(\mathrm{mg} \mathrm{kg}^{-1}\right)\end{array}$} & \multicolumn{2}{|c|}{$\begin{array}{c}\text { Lattice K } \\
(\%)\end{array}$} & \multicolumn{2}{|c|}{$\begin{array}{c}\text { Total K } \\
(\%)\end{array}$} \\
\hline & $\begin{array}{l}\text { Surface } \\
(0-20 \mathrm{~cm})\end{array}$ & $\begin{array}{c}\text { Sub } \\
\text { surface } \\
(20-50 \\
\text { cm) }\end{array}$ & $\begin{array}{l}\text { Surface } \\
(0-20 \mathrm{~cm})\end{array}$ & $\begin{array}{c}\text { Sub } \\
\text { surface } \\
(20-50 \mathrm{~cm})\end{array}$ & $\begin{array}{l}\text { Surface } \\
(0-20 \mathrm{~cm})\end{array}$ & $\begin{array}{c}\text { Sub } \\
\text { surface } \\
(20-50 \\
\text { cm) } \\
\end{array}$ & $\begin{array}{c}\text { surface } \\
(0-20 \\
\text { cm) }\end{array}$ & $\begin{array}{c}\text { Sub } \\
\text { Surface } \\
(20-50 \mathrm{~cm})\end{array}$ & $\begin{array}{l}\text { Isurface } \\
(0-20 \mathrm{~cm})\end{array}$ & $\begin{array}{c}\text { Sub } \\
\text { surface } \\
(20-50 \\
\mathrm{cm}) \\
\end{array}$ \\
\hline 1 & 3.24 & 2.82 & 45.81 & 35.60 & 540.96 & 690.31 & 0.92 & 0.98 & 0.98 & 1.05 \\
\hline 2 & 3.37 & 1.42 & 56.24 & 48.12 & 586.04 & 726.27 & 0.94 & 1.07 & 1.00 & 1.15 \\
\hline 3 & 2.02 & 1.83 & 45.86 & 36.61 & 608.58 & 690.29 & 1.04 & 1.15 & 1.10 & 1.23 \\
\hline 4 & 3.16 & 3.02 & 44.87 & 34.23 & 540.96 & 667.94 & 0.97 & 1.08 & 1.03 & 1.15 \\
\hline 5 & 1.71 & 1.31 & 48.70 & 41.60 & 586.04 & 756.44 & 1.04 & 1.12 & 1.10 & 1.20 \\
\hline 6 & 2.89 & 1.70 & 45.84 & 40.24 & 586.04 & 780.95 & 0.93 & 1.02 & 0.97 & 1.10 \\
\hline 7 & 4.69 & 3.39 & 68.40 & 56.09 & 540.96 & 622.17 & 1.25 & 1.38 & 1.30 & 1.45 \\
\hline 8 & 1.90 & 2.30 & 54.20 & 38.80 & 495.88 & 640.55 & 0.95 & 1.03 & 1.00 & 1.10 \\
\hline 9 & 3.19 & 3.16 & 44.82 & 39.14 & 518.42 & 592.27 & 1.15 & 1.26 & 1.20 & 1.32 \\
\hline 10 & 4.58 & 2.63 & 56.20 & 50.89 & 631.12 & 792.91 & 1.18 & 1.29 & 1.25 & 1.37 \\
\hline 11 & 2.00 & 2.06 & 44.80 & 30.60 & 653.66 & 860.85 & 1.25 & 1.38 & 1.33 & 1.47 \\
\hline 12 & 2.60 & 2.17 & 78.40 & 67.20 & 653.66 & 777.06 & 1.28 & 1.42 & 1.35 & 1.50 \\
\hline 13 & 2.96 & 1.57 & 52.11 & 56.11 & 631.12 & 765.21 & 1.06 & 1.14 & 1.13 & 1.23 \\
\hline 14 & 2.17 & 1.81 & 51.27 & 44.98 & 540.96 & 658.40 & 1.02 & 1.10 & 1.08 & 1.17 \\
\hline 15 & 2.32 & 1.63 & 54.81 & 49.62 & 518.42 & 630.40 & 1.09 & 1.20 & 1.15 & 1.27 \\
\hline 16 & 3.66 & 2.38 & 51.80 & 47.81 & 631.12 & 843.32 & 1.21 & 1.29 & 1.28 & 1.37 \\
\hline 17 & 1.66 & 1.44 & 48.80 & 42.60 & 495.88 & 637.61 & 1.15 & 1.26 & 1.20 & 1.33 \\
\hline 18 & 3.36 & 2.73 & 56.00 & 55.14 & 608.58 & 741.48 & 1.24 & 1.34 & 1.30 & 1.42 \\
\hline 19 & 4.02 & 3.06 & 49.01 & 45.89 & 631.12 & 821.02 & 1.21 & 1.33 & 1.28 & 1.42 \\
\hline 20 & 2.61 & 2.32 & 47.82 & 43.63 & 631.12 & 776.94 & 1.18 & 1.29 & 1.25 & 1.38 \\
\hline Range & $\begin{array}{l}1.66- \\
4.69\end{array}$ & $\begin{array}{l}1.31- \\
3.39\end{array}$ & $\begin{array}{l}44.80- \\
78.40\end{array}$ & $\begin{array}{l}30.60- \\
67.20\end{array}$ & $\begin{array}{c}495.88- \\
653.66\end{array}$ & $\begin{array}{l}592.27- \\
860.85\end{array}$ & $\begin{array}{l}0.92- \\
1.28\end{array}$ & $\begin{array}{l}0.98- \\
1.42\end{array}$ & $\begin{array}{l}0.97- \\
1.35\end{array}$ & $\begin{array}{l}1.05- \\
1.50\end{array}$ \\
\hline Mean & 2.91 & 2.24 & 52.28 & 45.24 & 581.53 & 723.62 & 1.11 & 1.20 & 1.16 & 1.28 \\
\hline S.D & 0.89 & 0.64 & 8.41 & 8.92 & 53.03 & 79.36 & 1.18 & 1.35 & 1.26 & 1.38 \\
\hline
\end{tabular}


Table.8 Forms and distribution of potassium in black soil type of selected maize growing areas of Haveri district

\begin{tabular}{|c|c|c|c|c|c|c|c|c|c|c|}
\hline $\begin{array}{c}\text { Sample } \\
\text { No. }\end{array}$ & \multicolumn{2}{|c|}{$\begin{array}{c}\text { Water soluble } \mathrm{K} \\
\left(\mathrm{mg} \mathrm{kg}^{-1}\right)\end{array}$} & \multicolumn{2}{|c|}{$\begin{array}{c}\text { Exchangeable } \mathrm{K} \\
\left(\mathrm{mg} \mathrm{kg}^{-1}\right)\end{array}$} & \multicolumn{2}{|c|}{$\begin{array}{c}\text { Non-Exchangeable K } \\
\left(\mathrm{mg} \mathrm{kg}^{-1}\right)\end{array}$} & \multicolumn{2}{|c|}{$\begin{array}{c}\text { Lattice K } \\
(\%)\end{array}$} & \multicolumn{2}{|c|}{$\begin{array}{c}\text { Total K } \\
(\%)\end{array}$} \\
\hline 1 & 4.60 & 3.67 & 56.00 & 44.81 & 620.67 & 743.90 & 1.38 & 1.53 & 1.45 & 1.60 \\
\hline 2 & 4.96 & 2.10 & 51.00 & 43.66 & 566.67 & 622.17 & 1.59 & 1.69 & 1.65 & 1.78 \\
\hline 5 & 3.40 & 2.34 & 56.01 & 48.27 & 619.24 & 829.06 & 1.61 & 1.69 & 1.68 & 1.78 \\
\hline 6 & 4.48 & 3.23 & 54.80 & 43.62 & 665.67 & 740.21 & 1.71 & 1.79 & 1.78 & 1.88 \\
\hline 7 & 4.79 & 3.43 & 67.25 & 53.60 & 621.30 & 769.48 & 1.66 & 1.77 & 1.73 & 1.85 \\
\hline 8 & 3.91 & 2.00 & 78.42 & 56.00 & 678.91 & 741.66 & 1.83 & 1.90 & 1.90 & 1.98 \\
\hline 9 & 2.83 & 2.26 & 48.92 & 36.90 & 646.38 & 719.39 & 1.63 & 1.76 & 1.70 & 1.83 \\
\hline 10 & 4.64 & 3.74 & 56.04 & 49.60 & 566.37 & 639.87 & 1.56 & 1.64 & 1.63 & 1.73 \\
\hline 15 & 8.13 & 4.91 & 89.60 & 67.20 & 673.15 & 817.49 & 1.96 & 2.02 & 2.03 & 2.10 \\
\hline 16 & 6.58 & 2.17 & 100.96 & 76.40 & 766.02 & 798.65 & 2.03 & 2.04 & 2.10 & 2.13 \\
\hline 17 & 7.20 & 3.00 & 67.20 & 56.00 & 726.35 & 804.40 & 1.90 & 2.02 & 1.98 & 2.10 \\
\hline 18 & 5.78 & 3.78 & 56.21 & 56.05 & 722.12 & 724.02 & 1.68 & 1.84 & 1.75 & 1.93 \\
\hline 19 & 7.31 & 5.45 & 67.20 & 54.80 & 703.65 & 754.25 & 1.68 & 1.78 & 1.75 & 1.87 \\
\hline 20 & 7.84 & 6.23 & 100.33 & 78.40 & 790.84 & 865.43 & 2.00 & 2.07 & 2.08 & 2.15 \\
\hline Range & 2.83-8.13 & $2.00-6.23$ & $\begin{array}{l}48.92- \\
100.96\end{array}$ & $\begin{array}{c}36.90- \\
78.40\end{array}$ & $\begin{array}{l}566.37- \\
790.84\end{array}$ & $\begin{array}{l}622.17- \\
865.43\end{array}$ & $1.38-2.03$ & $1.53-2.07$ & $\begin{array}{l}1.45- \\
2.10\end{array}$ & $1.60-2.15$ \\
\hline Mean & 4.60 & 3.67 & 56.00 & 46.81 & 686.49 & 773.34 & 1.72 & 1.82 & 1.79 & 1.90 \\
\hline S.D & 1.61 & 1.32 & 15.90 & 11.06 & 66.26 & 67.30 & 0.17 & 0.15 & 0.17 & 0.15 \\
\hline
\end{tabular}


Table.9 Correlation coefficients of different forms of $\mathrm{K}$ and selected soil properties in red soil type of maize growing areas of Haveri district

\begin{tabular}{|c|c|c|c|c|c|c|c|}
\hline \multirow{2}{*}{\begin{tabular}{c} 
Forms of potassium \\
\cline { 2 - 7 }
\end{tabular}} & $\mathbf{p H}$ & EC & OC & Sand & Silt & Clay & CEC \\
\cline { 2 - 7 } & 0.460 & -0.078 & 0.352 & $0.755^{*}$ & 0.345 & 0.227 \\
\hline Water soluble K & 0.496 & 0.161 & 0.378 & -0.231 & 0.388 & 0.489 \\
\hline Nonchangeable K & -0.297 & 0.266 & -0.020 & $-0.683^{* *}$ & 0.239 & $0.721 *$ \\
\hline Lattice K & -0.293 & 0.100 & -0.250 & -0.443 & $0.502^{* *}$ & $0.813^{*}$ & 0.422 \\
\hline Total K & 0.310 & 0.106 & 0.248 & -0.432 & 0.397 & $0.894^{*}$ & 0.412 \\
\hline
\end{tabular}

**Significant at $1 \%$ level of significance; * Significant at $5 \%$ level of significance

Table.10 Correlation coefficients of different forms of $\mathrm{K}$ and selected soil properties in black soil type of maize growing areas of Haveri district

\begin{tabular}{|c|c|c|c|c|c|c|c|}
\hline \multirow{2}{*}{ Forms of potassium } & \multicolumn{7}{|c|}{ Correlation coefficients (r values) } \\
\hline & pH & EC & OC & Sand & Silt & Clay & CEC \\
\hline Water soluble K & 0.235 & -0.233 & 0.487 & $0.527^{*}$ & 0.442 & 0.260 & 0.369 \\
\hline Exchangeable $\mathrm{K}$ & 0.283 & 0.218 & 0.391 & $-0.513^{*}$ & 0.454 & $0.563^{*}$ & 0.807 \\
\hline Non-exchangeable $\mathbf{K}$ & 0.252 & 0.287 & -0.147 & $-0.559 * *$ & 0.363 & $0.556^{*}$ & 0.259 \\
\hline Lattice $\mathbf{K}$ & -0.284 & 0.199 & -0.314 & -0.365 & $0.510 * *$ & $0.959 *$ & 0.354 \\
\hline Total K & 0.292 & 0.203 & $0.514 * *$ & -0.371 & $0.517 * *$ & $0.965^{*}$ & 0.358 \\
\hline
\end{tabular}

\footnotetext{
**Significant at $1 \%$ level of significance; * Significant at $5 \%$ level of significance
} 
Fig.1 Water soluble and exchangeable potassium in Red and Black soils of selected maize growing areas of Haveri district
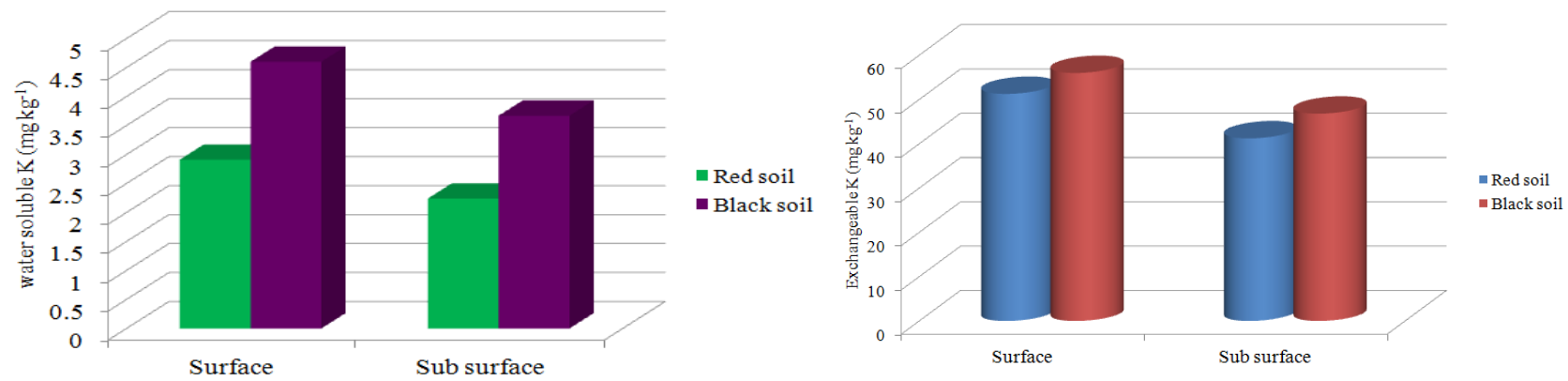

Fig.2 Non-exchangeable and lattice potassium in Red and Black soils of selected maize growing areas of Haveri district
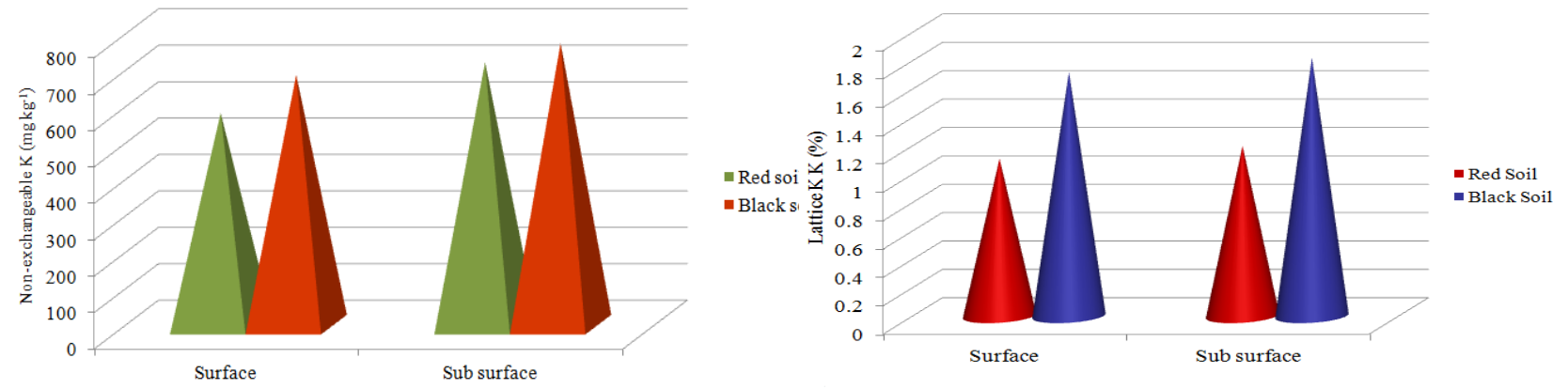

Fig.3 Total potassium and its distribution in red and black soils of selected maize growing areas of Haveri district

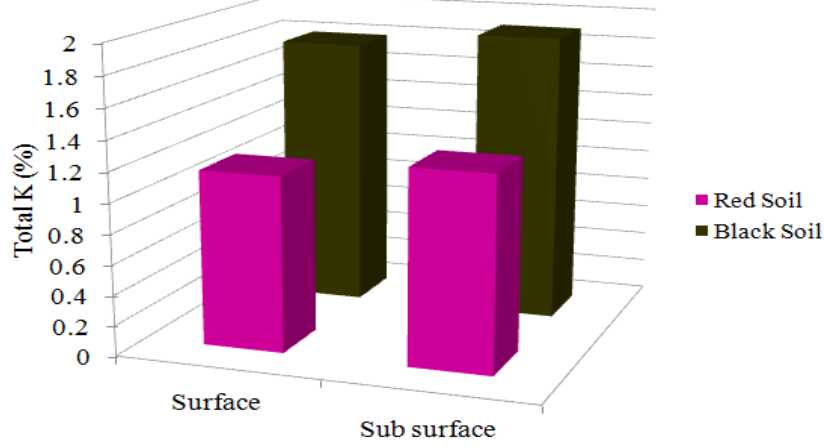

The average non-exchangeable potassium of surface layer in black soil type recorded 686.49 $\mathrm{mg} \mathrm{kg} \mathrm{k}^{-1}$ and that of corresponding sub surface recorded $773.34 \mathrm{mg} \mathrm{kg}^{-1}$. The non-exchangeable $\mathrm{K}$ was low in surface might be due to release of potassium from reserve pool to compensate the loss of water soluble and exchangeable $\mathrm{K}$ by plant uptake. The increase in non-exchangeable

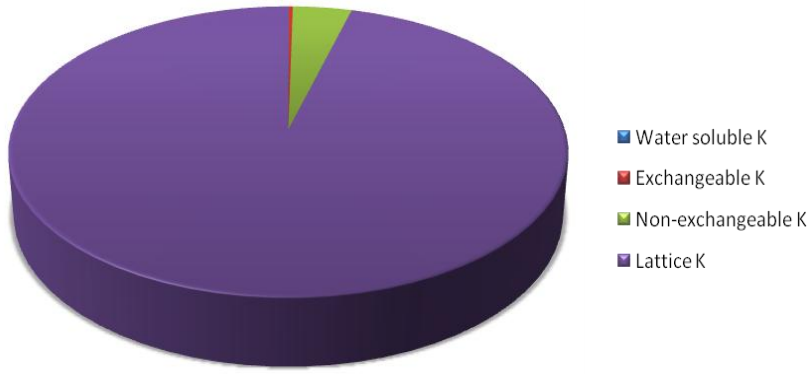

potassium at sub surface may be attributed to adsorption and fixation of $\mathrm{K}$ removed from surface through leaching. The similar findings were obtained by Kundu et al., (2014) and Divya et al., (2016). However, the nonexchangeable $\mathrm{K}$ content of black soil was more compared to red soils at both the depths may be because of nature and amount of clay present in 
black soils (Hebsur and Gali, 2011 and Kundu et al., 2014).

\section{Lattice potassium}

The mean values of lattice $\mathrm{K}$ in black soils was high (1.72 and 1.82 per cent in surface and subsurface samples, respectively) compared to red soils (1.11 and 1.20 per cent in surface and subsurface samples, respectively). The higher values of sub surface indicate that these soils have been derived from potassium bearing minerals such as 2:1 type of clay minerals which favoured the lattice potassium content in soils. Based on degree of weathering and soil type the surface and sub-surface lattice $\mathrm{K}$ content might have been varied among the samples. The results corroborated with the findings of Divya et al., (2016) (Fig. 2).

\section{Total K}

The average values of total $\mathrm{K}$ was high in black soils (1.79 and 1.90 per cent in surface and subsurface samples, respectively) compared to red soils (1.16 and 1.28 per cent in surface and subsurface samples, respectively). The total $\mathrm{K}$ followed a trend of increase with depth. The black soils were rich with average total potassium compared to red soils irrespective of depth. It may be because of the rich K-bearing minerals in their lattice structure (Anil et al., 2009). Depending on clay mineralogy, lattice $K$ content and organic matter content, the total $\mathrm{K}$ content might have been varied in surface and sub-surface layers. The results were in comparison with those of research findings of Gali and Hebsur (2011), Jagmohan and Grewal (2014) and Divya et al., (2016) (Fig. 3).

\section{Correlation studies}

\section{Correlation (r-values) studies among different forms of potassium and soil properties}

The correlation values for different soil properties and soil $\mathrm{K}$ forms in selected soil types are presented in tables 9 and 10 , respectively.

In both red and black soil types, the water soluble $\mathrm{K}$ showed a positive correlation with sand $\left(\mathrm{r}=0.755^{*}\right.$ and $\left.0.527 *\right)$. It may be due to fact that $\mathrm{K}$ ion present on sand particle can come easily into solution. The exchangeable $\mathrm{K}$ was positively and significantly correlated with clay it may be because of higher content of $\mathrm{K}$ bearing minerals present which might be having higher exchangeable surface.

The exchangeable $\mathrm{K}$ showed a positive correlation CEC and $\mathrm{pH}$ which may due be due to fact that increases in $\mathrm{pH}$ results in increase in CEC, which in turn might have increased exchangeable $\mathrm{K}$. The non-exchangeable $\mathrm{K}$ was significantly and positively correlated with clay $(\mathrm{r}=0.868 * \& 0.556 *)$ as clays have greater ability to fix $\mathrm{K}$ in non-exchangeable form. The sand fraction was negatively and significantly correlated with non-exchangeable K. It may be due to fact that sand particles lack fixation sites because of their coarse nature.

The lattice $\mathrm{K}$ was significantly and positively correlated with clay and silt which may be due to presence of $\mathrm{K}$ bearing minerals in clay and silt fractions. Similar trend followed for total K as that of lattice $K$ as much of lattice $K$ is contributed to total $\mathrm{K}$ and finer fractions of soils rich in $\mathrm{K}$-bearing minerals as it is evident from the correlation coefficients. The present results were in agreement with the findings of Jagadeesh (2003), Kundu et al., (2014), Jagmohan and Grewal (2014) and Hemlata (2016).

The results of the investigation suggested that in the Haveri district of Karnataka, maximum $\mathrm{K}$ content of the soils is in the non-exchangeable form, mostly fixed up within the clay lattice rendering very small amount of available $\mathrm{K}$ to plant. The forms of potassium were significantly correlated among different physicchemical properties. Knowledge of different forms of potassium in soil together with their distribution has greater relevance in assessing the long-term $\mathrm{K}$ supplying power of soil to crops and is important in formulating a sound fertilizer programme for a given set of soil and 
crop. A future study on clay mineralogical make-up may help in calibrating the reserve pool of $\mathrm{K}$ and the extent of its mining. This helps the planners to formulate an effective $\mathrm{K}$ fertilizer programme for the soils of the region.

\section{References}

Anil, S., Jalali, V. K., Arya, V. M. and Pradeep, R., 2009. Distribution of various forms of potassium in soils representing intermediate zone of Jammu region. $J$. Ind. Soc. Soil Sci., 57(2): 205-207.

Black, C.A., 1965. Methods of Soil Analysis Part- II. Chemical and mineralogical properties. Agronomy Monograph No. 9, Am. Soc. Agron., Inc. Madison, Wisconsin, USA, pp. 18-25.

Britzke, D., Silva, L. S., Moterle, D. F., Rheinheimer, D. and Bortoluzzi, E. C., 2012. A study of potassium dynamics and mineralogy in soils from subtropical Brazilian lowlands. J. Soils Sediments, 12: $185-197$.

Datta, S.C., 2011. Potassium dynamics and status in Indian soils. Karnataka J. Agric Sci., 24(1): 7-11.

Divya, M., Jagadeesh, B. R., Srinivasa, D. K. and Yogesh, G. S., 2016. Effect of long term soil fertilizer application on forms and distribution of potassium in soil under rice-cowpea cropping system. An Asian J. Soil Sci., 11(1): 10-19.

Hebsur, N.S., and Gali, S. K., 2011. Potassium Dynamics in Soils under different cropping systems of Karnataka. Soil Sci. Res. North Karnataka, 76th Annual Convention Indian Soc. Soil Sci., 85-89.

Hemlata, D., 2016. Status of different forms of potassium in soils of Gwalior district (M.P.). M.Sc. (Agri.) Thesis, Rajmata Vijayaraje Scindia Krishi Vishwa
Vidyalaya, College of Agriculture, Gwalior (M. P.).

Jackson, M.L., 1973, Soil Chemical Analysis, Prentice Hall of India Private Limited, New Delhi pp. 132-148.

Jagadeesh, B.R., 2003. Dynamics of potassium in soils of selected agro- climatic zones of Karnataka. Ph.D. Thesis, Univ. Agric. Sci., Bangalore, Karnataka (India).

Jagmohan, S. and Grewal, K. S., 2014. Vertical distribution of different forms of potassium and their relationship with different soil properties in some Haryana soil under different crop rotation. $A d v$. Plant Agricl. Res., 1(2): 1-5.

Knudsen, D., Peterson, G. J. and Pratt, P. F., 1982. Lithium, sodium and potassium. In methods of soil analysis part IIChemical and Microbiological properties. Ed. page, A.L., American society of Agronomy, Inc., Soil Sci. Soc. Am. Inc. Madison, Wisconsin, USA. pp. 147-151.

Kundu, M.C., Hazra, G. C., Biswas, P. K., Mondal, S. and Ghosh, G. K., 2014. Forms and distribution of potassium in some soils of Hooghly district of West Bengal. J. Crop Weed, 10(2): 31-37.

Lim, C. H., and Jackson, M. L., 1982. Dissolution for total elemental analysis In: Methods of soil analysis Part II. Chemical and Microbiological properties. Ed. page, A.L., American society of Agronomy, Inc., Soil Sci. Soc. Am. Inc., Madison, Wisconsin, USA. pp. 192-196.

Piper, C. S., 1996. Soil and Plant Analysis. Hans Publishers, Bombay, India. pp. 3352.

U. S. D. A. Handbook 60, 1954. Diagnosis and Improvement of Saline and Alkali Soils. U.S. Government Printing Office, Washington D. C., pp. 22-26.

\section{How to cite this article:}

Harsha, B.R. and Jagadeesh, B.R. 2017. Forms and Distribution of Potassium in Selected Maize Growing Soils of Haveri District of Karnataka. Int.J.Curr.Microbiol.App.Sci. 6(9): 923-936. doi: https://doi.org/10.20546/ijcmas.2017.609.111 